\title{
Architected poly(lactic acid)/poly(e-caprolactone)/ halloysite nanotube composite scaffolds enabled by 3D printing for biomedical applications
}

\author{
Fahad Alam ${ }^{1}$, Pawan Verma ${ }^{1,2}$, Walaa Mohammad $^{3}$, Jeremy Teo $^{3}$, K. M. Varadarajan ${ }^{4,5}$, and \\ S. Kumar ${ }^{1,6, *}$ \\ ${ }^{1}$ Department of Mechanical Engineering, Khalifa University of Science and Technology, Masdar Campus, P.O. Box 54224, Mas- \\ dar City, Abu Dhabi, UAE \\ ${ }^{2}$ Artie McFerrin Department of Chemical Engineering, Texas A\&M University, College Station, TX 77840, USA \\ ${ }^{3}$ Division of Engineering, New York University Abu Dhabi, P.O. Box 129188, Abu Dhabi, UAE \\ ${ }^{4}$ Department of Orthopaedic Surgery, Harris Orthopaedics Laboratory, Massachusetts General Hospital, 55 Fruit St, Boston, USA \\ ${ }^{5}$ Department of Orthopaedic Surgery, Harvard Medical School, A-111, 25 Shattuck Street, Boston, USA \\ ${ }^{6}$ James Watt School of Engineering, University of Glasgow, Glasgow G12 8LT, UK
}

Received: 15 December 2020

Accepted: 4 May 2021

Published online:

27 May 2021

(C) The Author(s) 2021

\begin{abstract}
Herein, we report the physicochemical, thermal, mechanical and biological characteristics, including bioactivity, biodegradation and cytocompatibility of additive manufacturing-enabled novel nanocomposite scaffolds. The scaffolds comprise a blend of polylactic acid (PLA) and poly-e-caprolactone (PCL) reinforced with halloysite nanotubes (HNTs). The nanoengineered filaments were developed by melt blending, and the nanocomposite scaffolds were manufactured by fused filament fabrication. Uniform dispersion of HNTs in the PLA/PCL blend is revealed via scanning electron microscopy. Mechanical property loss due to the addition of PCL to realize a suitable biodegradation rate of PLA was fully recovered by the addition of HNTs. Bioactivity, as revealed by the fraction of apatite growth quantified from XRD analysis, was 5.4, 6.3, 6.8 and 7.1\% for PLA, 3, 5 and $7 \mathrm{wt} \%$ HNT in PLA/PCL blend, respectively, evidencing enhancement in the bioactivity. The degradation rate, in terms of weight loss, was reduced from $4.6 \%$ (PLA) to 1.3\% (PLA/PCL) upon addition of PCL, which gradually increased to $4.4 \%$ by the addition of HNTs (at 7 wt $\%$ HNT). The results suggest that the biodegradation rate, mechanical properties and biological characteristics, including cytocompatibility and cell adhesion, of the 3D printed, microarchitected PLA/PCL/HNT composite scaffolds can be tuned by an appropriate combination of HNT and PCL content in the PLA matrix, demonstrating their promise for bone replacement and regeneration applications.
\end{abstract}

Handling Editor: Chris Cornelius.

Address correspondence to E-mail: s.kumar@eng.oxon.org 


\section{GRAPHICAL ABSTRACT}
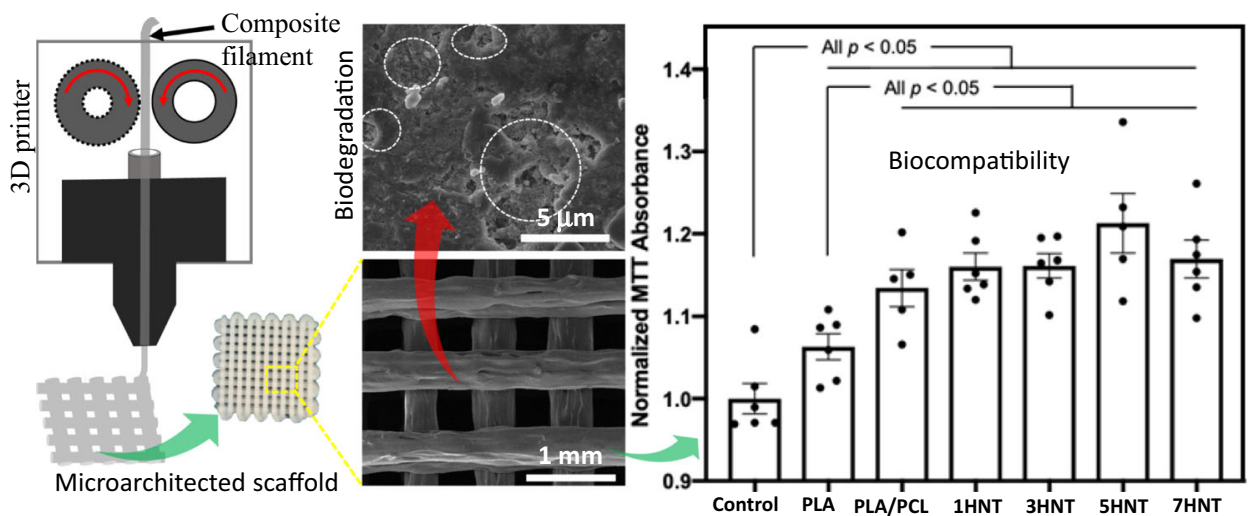

\section{Introduction}

Blends of polylactic acid (PLA) and poly- $\varepsilon$-caprolactone (PCL) are extensively being explored recently for biomedical applications due to their promising characteristics, including biodegradability. Both PLA and PCL are regarded as biomedical materials by Food and Drug Administration USA, due to their biocompatibility [1, 2]. The end-product obtained after biodegradation of PLA, such as water, carbon dioxide and hydroxyl acid, is well accepted by the human body [3, 4]. Although PLA has adequate strength, it lacks mechanical compliance [5-7]. Moreover, PLA exhibits poor wettability but shows a faster biodegradation rate as compared to PCL. Aiming to tailor desirable properties, researchers have utilized natural fibers such as silk, collagen and chitosan-based PLA composites. Blending of PLA with PCL is one of the alternative routes opted for controlling the degradation rate and other properties [8-11]. PCL has high strain-to-break with controlled carrier release properties but a slow degradation rate (up to 4 years) [12]. The blend of PLA and PCL is desired to achieve an optimal combination of biomedical and mechanical properties [13] and is therefore widely considered for tissue engineering applications. PLA offers better mechanical strength while PCL provides strain tolerance, enhanced permeability and reduced rate of degradation [14]. To improve the surface wettability of the scaffolds, different approaches such as post-fabrication surface modification by thin coating or by plasma treatment have been explored. Incorporation of nanomaterials such as carbon nanostructures, metal oxide nanoparticles into polymer matrix is yet another approach to alter the surface wettability [15]. Halloysite nanotube (HNT) has gained attraction in the recent past as a biocompatible material as it has the potential to enhance the biocompatibility [16, 17], as well as surface wettability of the polymer matrix [18-20]. HNT - a clay mineral belonging to kaolinite family-has a long tubule-like structure with a stoichiometry of $\mathrm{Al}_{2} \mathrm{Si}_{2} \mathrm{O}_{5}(\mathrm{OH}) 4 \cdot \mathrm{nH}_{2} \mathrm{O}$. A few studies were focused on 3D printing of PLA with HNT for biomedical applications [21-25]. However, the combination of PCL with PLA/HNT is not much explored.

The polymer composite scaffolds can be fabricated by various manufacturing techniques including phase separation, gas foaming and additive manufacturing (AM). Among these techniques, AM (also known as $3 \mathrm{D}$ printing) has emerged as one of the most suitable techniques for polymer scaffold fabrication as it offers design freedom [26-32]. Among various $3 \mathrm{D}$ printing techniques, fused deposition modeling (FDM), also known as fused filament fabrication (FFF), is a preferred method for the fabrication of polymeric scaffolds with microarchitected internal and external porous morphology in a controlled fashion $[33,34]$. In FFF 3D printing, a filament feedstock (diameter $\sim 1.75$ or $2.4 \mathrm{~mm}$ ) is fed into the 
heated nozzle and the resulting melt is deposited on the preheated print bed in a layer-by-layer fashion, forming a 3D structure. FFF is relatively easy, fast and cost-effective 3D printing technique [35-38].

In this study, the PLA/PCL/HNT composite filaments were developed by melt extrusion and composite scaffolds with porosity, $\emptyset=50 \%$ (conversely, relative density, $\bar{\rho}=(1-\emptyset)=50 \%)$, were fabricated via FFF 3D printing. Both PLA and PCL were mixed in 1:1 ratio and the HNT content was varied from 1 to $7 \mathrm{wt} \%$ in PLA/PCL blend. The physicochemical, thermal and mechanical characteristics of the scaffolds were analyzed. In vitro bioactivity, biodegradation and cytocompatibility of the composite scaffolds were also characterized to access their potential for bone replacement and regeneration applications.

\section{Experimental}

\section{Materials}

The pellets of PLA (3D850) obtained from NatureWorks ${ }^{\circledR}$ LLC and were used for filament fabrication. PCL was supplied by Sigma-Aldrich (product number 440744) and has a molecular weight of $\sim 80000$ $\mathrm{g} / \mathrm{mol}$ and a density of $1.14 \mathrm{~g} / \mathrm{cm}^{3}$. HNT used in this study was supplied by Sigma-Aldrich and has an average outer diameter of $\sim 50 \mathrm{~nm}$ (see, TEM image shown in Fig. 1). All other chemicals used to prepare simulated body fluid (SBF) and other biological assays were of analytical or higher grade. Table 1 summarizes different compositions of PLA, PCL and

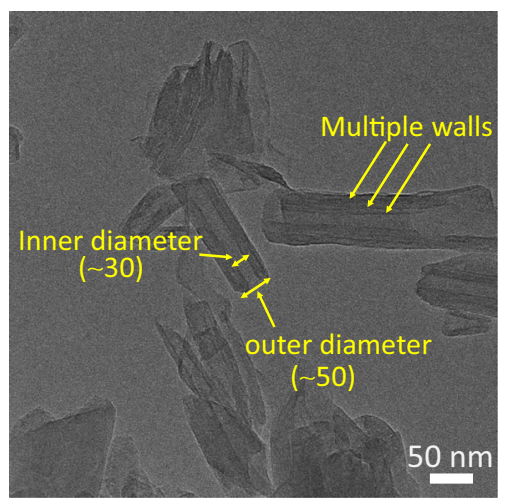

Figure 1 TEM image of the HNTs used in the current study.
Table 1 Compositions of the filaments developed by melt blending

\begin{tabular}{lrcl}
\hline Sample ID & PLA (wt $\%)$ & PCL (wt $\%)$ & HNT (wt\%) \\
\hline PLA & 100 & 0 & 0 \\
PLA/PCL & 50 & 50 & 0 \\
1HNT & 50 & 50 & 1 \\
3HNT & 50 & 50 & 3 \\
5HNT & 50 & 50 & 5 \\
7HNT & 50 & 50 & 7 \\
\hline
\end{tabular}

HNT used in this study and their sample identification.

\section{Filament fabrication and 3D printing of PLA/PCL blend and PLA/PCL/HNT composites}

The schematic illustration of filament fabrication and $3 \mathrm{D}$ printing is shown in Fig. 2. PLA and PCL were mixed in 1:1 ratio and the HNT content was varied from 1 to $7 \mathrm{wt} \%$ in PLA/PCL blend. Dichloromethane (DCM) was used as a solvent. All the constituents were mixed with a magnetic stirrer and the slurry made was dried by solvent evaporation in the form of a sheet. The sheets were then chopped into small pieces (Fig. 2a (i)) and fed into the extruder (Fig. 2a (ii)). A twin-screw extruder was used to prepare the filament feedstock with a diameter of $1.75 \mathrm{~mm}$ as shown in Fig. 2a (iii). The fabricated filament was then collected and stored in spools. The developed filaments were used for 3D printing of test specimens.

The scaffolds of selected compositions were fabricated by FFF AM using a commercial 3D printer (Creater Pro 3D printer, Flashforge Cooperation Zhejian, China). The schematic of $3 \mathrm{D}$ printing is shown in Fig. 2a (iii). A Simplify3D software was used to generate the G Code-a file required for the FFF printer, using the STL file of the computer aided design (CAD) model. The relative density of the scaffold was controlled by appropriately architecting the pores in CAD model. For a designed relative density of $50 \%$, the pore diameter was in the range of 300-600 $\mu \mathrm{m}$. The developed filaments of $\sim 1.75 \mathrm{~mm}$ diameter were mounted directly on print head as shown in Fig. 2 to achieve high extrusion pressure 
Figure 2 a Schematic illustration of filament fabrication and $3 \mathrm{D}$ printing of the samples via FFF additive manufacturing. The process involves (i) solvent casting followed by (ii) extrusion to obtain filaments and finally (iii) 3D printing of scaffolds. b Optical images of the samples prepared for physicochemical characterization and (c) for cell culture test: (i) neat PLA, (ii) PLA/PCL, (iii) 1HNT, (iv) $3 \mathrm{HNT}$, (v) 5HNT and (vi) 7HNT. The numerals 1, 3, 5 and 7 refer to $w t \%$ of HNT in the samples. (a)

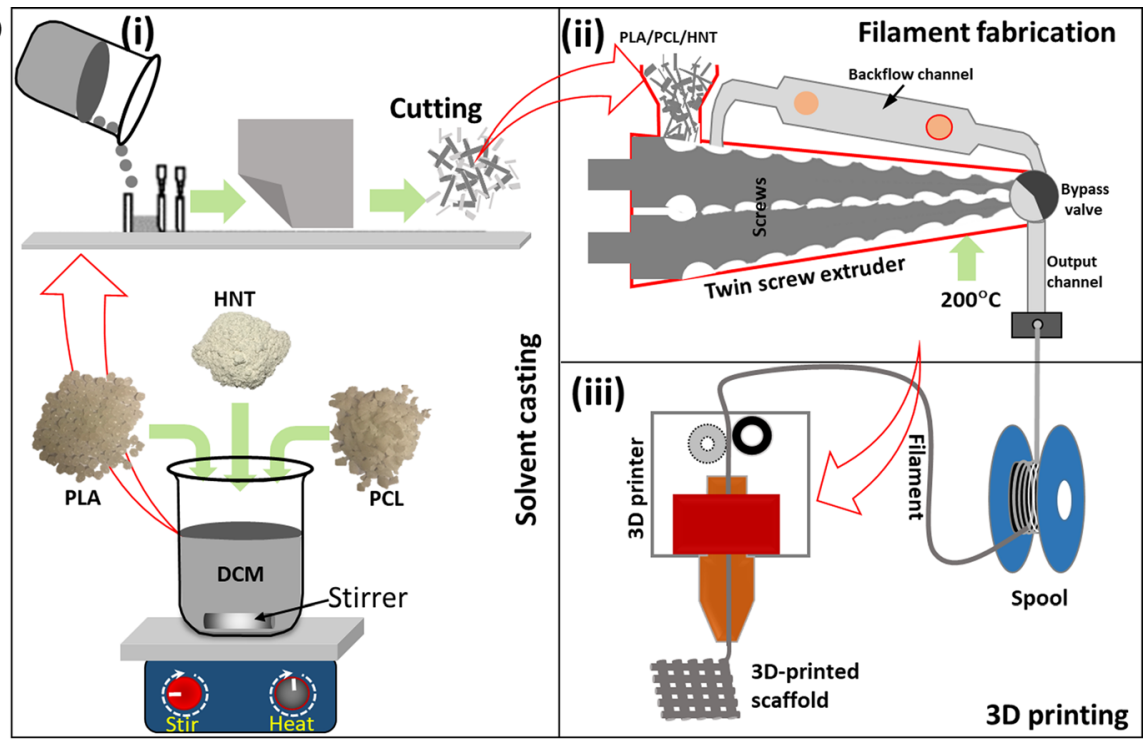

(b) (1)

(ii)

(iii)

(iv)

(v)

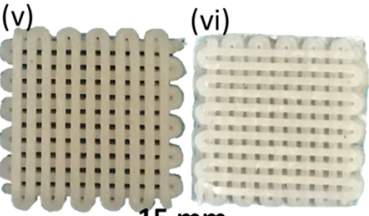

(c) (i)

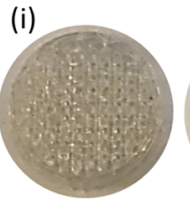

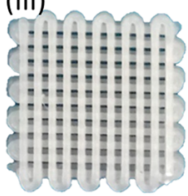
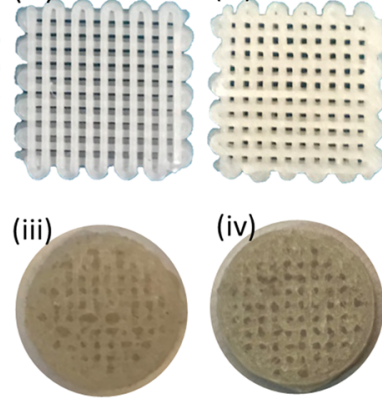
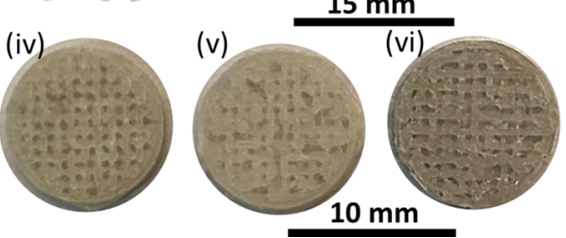

and avoid any problem in feeding. The specimens for physical, chemical, bioactivity and biodegradation tests were prepared in square shapes, whereas for cytotoxicity and adhesion tests, circular disks were prepared. The optical images of the prepared scaffolds are shown in Fig. $2 b$ and c. The parameters used in FFF 3D printing of scaffolds are presented in Table 2 .

Table 2 Process parameters used for FFF 3D printing

\begin{tabular}{ll}
\hline Parameters & Values \\
\hline Nozzle temperature & $220{ }^{\circ} \mathrm{C}$ \\
Print bed temperature & $60{ }^{\circ} \mathrm{C}$ \\
Nozzle speed & $1800 \mathrm{~mm} / \mathrm{min}$; first layer $300 \mathrm{~mm} / \mathrm{min}$ \\
Layer height & $0.1-0.3 \mathrm{~mm}$ \\
Extrusion width & $0.48 \mathrm{~mm}$ \\
Infill density & $50 \%$ \\
\hline
\end{tabular}

\section{Characterization}

\section{Physicochemical characterization}

The nanostructure of HNT used in this study was characterized via transmission electron microscopy (TEM) using FEI, Tecnai TEM 200 kV. TEM imaging was performed to confirm the nanostructure of the HNT. All the raw materials and in-house developed filaments were characterized by $X$-ray diffraction (XRD) to determine the compositions of all the constituents present. The XRD analysis was performed on a PAN analytical Empyrean diffractometer utilizing a $\mathrm{Cu}, K_{\alpha}$ radiation $(\lambda=1.54 \AA)$ operated at $40 \mathrm{kV}$, as an X-ray source. The samples were scanned at a speed of $2.4^{\circ} / \mathrm{min}$ with a step size of $0.02^{\circ}$ with $2 \theta^{\circ}$ ranging from 10 to $80^{\circ}$. The surface of samples after bioactivity tests was also analyzed using XRD to confirm the presence of apatite layer.

The surface topography and internal morphology of the filaments, as well as the 3D printed scaffolds, were observed under scanning electron microscopy 
(SEM). The FEI Nova NanoSEM 650 was utilized to obtain micrographs. The SEM was operated under an accelerating voltage of $5 \mathrm{kV}$ with a working distance of $5 \mathrm{~mm}$ and a spot size of $2.5 \mathrm{~nm}$. Prior to imaging under SEM, the surface of the samples was coated with a thin layer $(\sim 10 \mathrm{~nm})$ of $\mathrm{Au} / \mathrm{Pt}$ to make the surface conducting. The internal morphology of the filaments was observed on cryogenically fractured (brittle fracture under Liquid nitrogen) surfaces of the samples.

The thermal properties of the filaments were assessed via differential scanning calorimetry (DSC) and thermogravimetric analysis (TGA). DSC analysis was performed on a DSC 404, F1 (NETZSCH hightemperature DSC). The tests were performed under inert atmosphere (a flux of $20 \mathrm{ml} / \mathrm{min}$ ). The pinch (10-15 mg) of samples was subjected to heating scan ranging from $30-300{ }^{\circ} \mathrm{C}$, at a heating rate of $10{ }^{\circ} \mathrm{C} /$ $\min$. The glass transition temperature $\left(T_{\mathrm{g}}\right)$ and melting temperature $\left(T_{\mathrm{m}}\right)$ were evaluated from the obtained DSC data. The TGA was performed utilizing a STA 449 F3 (NETZSCH high-temperature TGA) instrument in the presence of inert atmosphere (a flux of $20 \mathrm{ml} / \mathrm{min}$ ). A small quantity of samples ( $15 \mathrm{mg}$ ) was scanned ranging from 30 to $600{ }^{\circ} \mathrm{C}$. The amount of solid residue was quantified to confirm the filler contents.

The surface wettability of the 3D printed samples was evaluated using water contact angle measurements. A contact angle goniometer (Krüss $\mathrm{GmbH}$ Drop shape Analyzer, DSA, Germany) was used for this purpose. A droplet of $5 \mu \mathrm{l}$ of deionized (DI) water was dropped on the surfaces. The static mode of sessile drop method was utilized to make the droplet and contact angle was recorded using a tangent method. At least three droplets were formed on each surface at different locations and the average values were reported.

The mechanical properties of the solvent cast dogbone samples were examined using a ZwickRoell Z005 universal testing machine (UTM) at a constant crosshead speed of $5 \mathrm{~mm} / \mathrm{min}$ at ambient temperature with a load cell of $2.5 \mathrm{kN}$ as per ASTM D638. The modulus, tensile strength and elongation were calculated from the stress-strain plots obtained. The maximum stress observed on the samples was reported as tensile strength $(\sigma)$ and the modulus is calculated from the initial linear portion of the stressstrain curve. Five specimens of each composition were tested and the average values with standard error are reported.

\section{Biological characterization}

Bioactivity of the scaffolds was evaluated by immersing samples in simulated body fluid (SBF) an artificial solution whose chemical composition is similar to human blood plasma, under incubation condition $\left(37^{\circ} \mathrm{C}\right)$ for an exposure time of $72 \mathrm{~h}$. The ions $\left(\mathrm{Ca}^{2+}\right.$ and $\left.\mathrm{HPO}_{4}^{2-}\right)$ present in SBF promote the growth of hydroxyapatite (also known as apatite). The samples were dried in oven at $50{ }^{\circ} \mathrm{C}$ overnight after immersion in SBF and apatite growth was examined via XRD analysis. The surface of apatitegrown scaffolds was further examined under SEM to observe the morphology of apatite growth.

In vitro degradation study of fabricated scaffolds was performed by immersing them in SBF. The scaffolds were immersed for different exposure times in $\mathrm{SBF}$ at $37^{\circ} \mathrm{C}$, for maintaining the same volume up to 30 days. The old SBF solution was replaced with fresh solution every week. The degradation was quantified by measuring the weight loss. The dry weight was recorded before and after immersion in SBF.

Fabricated scaffolds were ethanol sterilized, washed well with PBS three times and submerged in complete cell culture media (DMEM supplemented with 10\% FBS and 1\% Pen/Strep) for $24 \mathrm{~h}$. Scaffolds were then placed in Transwell cell culture plates with human dermal fibroblasts grown to confluence at the bottom, inhibiting contact between scaffolds and cells. Cells were left for $24 \mathrm{~h}$ and MTT was performed to determine if constituents leached from the scaffolds are toxic from the cells.

Subsequently, similarly treated scaffolds were placed on glass coverslips placed on conventional tissue culture plates and excess media was removed while keeping the material of the scaffold wetted. Untreated glass prevents the wicking away of cell culture suspension. $50 \mu \mathrm{L}$ of media containing $50 \mathrm{k}$ fibroblasts was placed on each scaffold and left for $4 \mathrm{~h}$. After which, wells were filled with $500 \mu \mathrm{L}$ of media and left to culture for $72 \mathrm{~h}$. After the duration of culture, scaffolds were removed using tweezers, placed into new cell culture plates and MTT was again performed to assess the number of cells on the scaffolds. Fluorescence imaging of fibroblasts stained 
with DAPI and Phalloidin was performed to representatively demonstrate the attachment of cells on the fabricated scaffolds.

\section{Results and discussion}

\section{Physical and thermal characteristics of filaments and 3D printed scaffolds}

The microstructural characterization of the filament feedstocks made of neat PLA, PLA/PCL blends and PLA/PCL/HNT composites was conducted using SEM. The SEM micrographs are shown in Fig. 3a-f. The filaments were cryogenically fractured to observe the cross section under SEM and diameter of all the filaments was in the range of $1.65-1.80 \mathrm{~mm}$. A small spot on the cross section was then magnified (highlighted by yellow dotted box) to observe the internal morphology of the filaments and assess the state of distribution of all three phases (PLA, PCL and HNT). The results were in close agreement with the SEM images reported in a recent study by Sluf et al. 2020 [39]. Figure $3 a$ and $b$ shows the surfaces of neat PLA and PLA/PCL blend, respectively. It can be observed from the figures that neat PLA exhibits a smooth and uniform surface morphology while PLA/PCL blend shows a less brittle fracture surface with two phases. The presence of HNT can be clearly observed in Fig. 3d-f. Dotted white circles indicate the presence of HNT in the samples. It is evident from the micrographs that the distribution density of HNT increased with increase in loading of HNT in PLA/ PCL matrix. Furthermore, a good dispersion of HNT in PLA/PCL matrix is clearly visible. At higher HNT loading, (such as at $5 \mathrm{wt} \% \mathrm{HNT}$ ), aggregation of HNT was observed to some extent and this was also evident from the thermal analysis.

The phases present in the filaments were characterized by XRD and the XRD spectra obtained are shown in Fig. 3g. The XRD spectra of PLA/PCL/ HNT composite were compared with those of neat PLA, PCL and HNT to identify the characteristic
Figure 3 Characterization of filaments: Microstructure of filaments (fabricated by melt extrusion process) obtained from SEM showing the internal features. a Neat PLA, b PLA/PCL blend, $\mathbf{c} 1 \mathrm{HNT}$ in PLA/PCL, $\mathbf{d} 3 \mathrm{HNT}$ in PLA/ PCL, e 5HNT in PLA/PCL and $\mathbf{f}$ 7HNT in PLA/PCL. The filaments were fractured cryogenically to observe under SEM. The magnified portion of microstructures is indicated by dotted yellow boxes.

Dotted white circles indicate the presence of HNT in PLA/ PCL blend. $g$ XRD spectra of the filaments, $\mathbf{h}$ DSC plots of the filaments and $\mathbf{i}$ TGA plots of the filaments compared with neat PLA, and PLA/PCL blend.
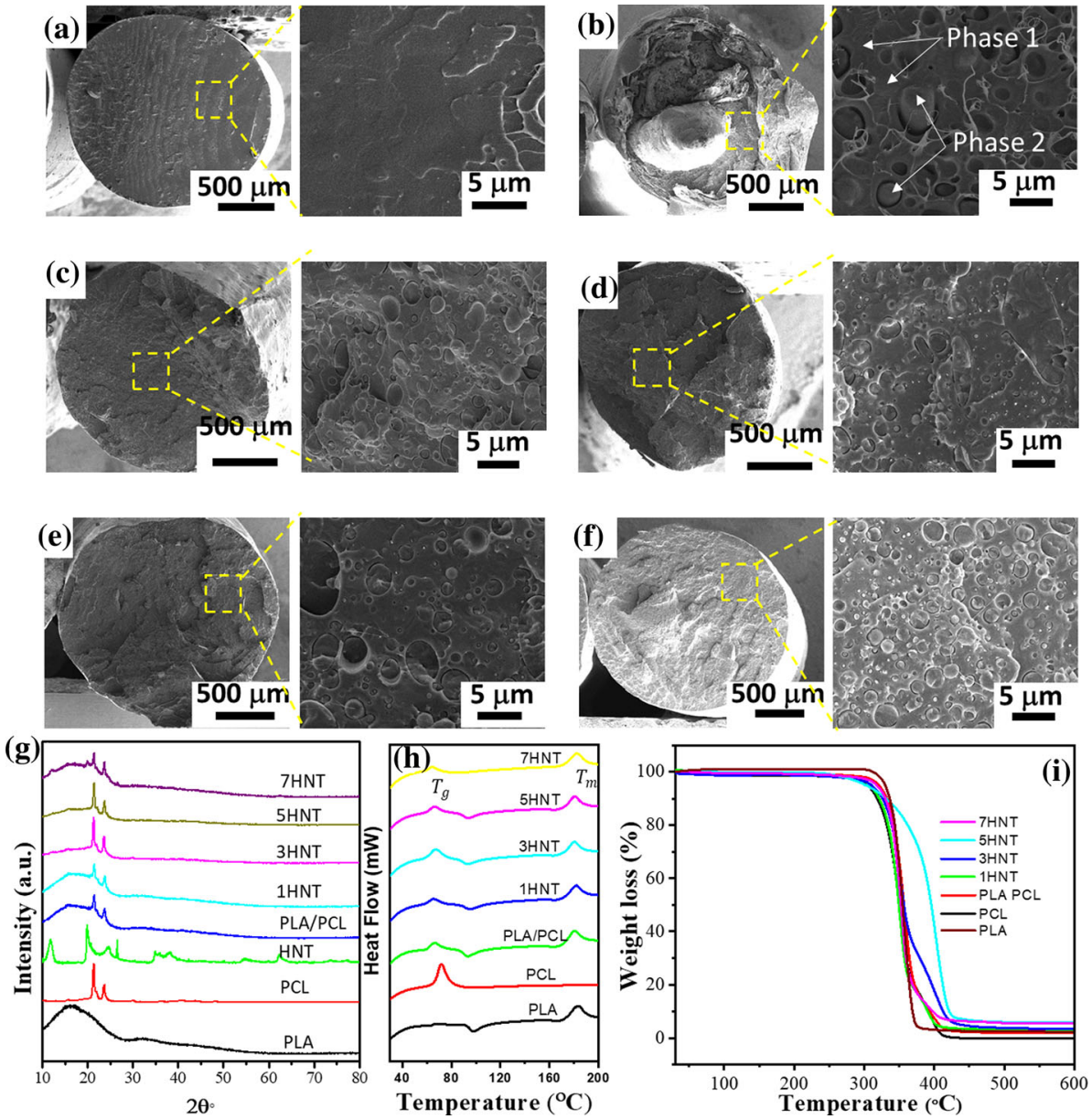
peaks. Neat PLA showed amorphous nature with a broad hump [40, 41], whereas PCL and HNT exhibited characteristic peaks showing semi-crystalline and crystalline nature, respectively [42]. The appearance of all peaks in PLA/PCL/HNT composite sample confirms the presence of all three constituents.

Thermal properties were characterized using DSC and TGA and the results are shown in Fig. $3 \mathrm{~h}$ and i, respectively. Glass transition temperature $T_{\mathrm{g}}$ and melting temperature $T_{\mathrm{m}}$ were evaluated from DSC analysis and the results are presented in Table 3. $T_{\mathrm{g}}$ decreased with the addition of PCL. However, no significant change in $T_{\mathrm{g}}$ was observed due to the addition of different wt $\%$ of HNT into PLA/PCL blend. $T_{\mathrm{g}}$ was reduced from $69.15{ }^{\circ} \mathrm{C}$ (neat PLA) to $66.59{ }^{\circ} \mathrm{C}$ (PLA/PCL) and $64.74{ }^{\circ} \mathrm{C}$ (7HNT) [43]. Marginal change in melting point was observed due to the addition of PCL into PLA but it was recovered to the level of neat PLA with the addition of HNT into PLA/PCL blend. Neat PLA exhibited a $T_{\mathrm{m}}$ of $\sim 183{ }^{\circ} \mathrm{C}$, which reduced to $\sim 180{ }^{\circ} \mathrm{C}$ upon addition of PCL into PLA and again recovered to $182{ }^{\circ} \mathrm{C}$ with the addition of $7 \mathrm{wt} \%$ HNT into PLA/ PCL blend. Though the change of melting temperature is irregular in case of $3 \mathrm{HNT}$, it is still within the experimental error of the TGA instrument. The fraction (wt\%) of HNT present in filaments developed was quantified from TGA analysis by measuring the amount of solid residue. The amount of residue reflects the amount of HNT loading. The plots of weight loss (\%) with respect to temperature are shown in Fig. 3i. The weight fraction of HNT was quantified from the solid residue and it was in line with the amount of the HNT added to PLA/PCL blend. However, the amount of residue for both 5 HNT and 7HNT samples is almost the same. This discrepancy might be attributed to the small amount of sample used for the TGA. To support the results,

Table 3 Thermal properties of the filaments obtained from DSC analysis

\begin{tabular}{lll}
\hline Samples ID & $T_{\mathrm{g}}\left({ }^{\circ} \mathrm{C}\right)$ & $T_{\mathrm{m}}\left({ }^{\circ} \mathrm{C}\right)$ \\
\hline PLA & 69.15 & 183.22 \\
PLA/PCL & 66.59 & 180.92 \\
1HNT & 65.36 & 181.71 \\
3HNT & 66.45 & 180.90 \\
5HNT & 65.79 & 181.12 \\
7HNT & 64.74 & 182.09 \\
\hline
\end{tabular}

SEM micrographs of cryogenically fractured samples were obtained.

The surface of as-prepared scaffolds was observed under SEM and the micrographs are shown in Fig. 4. The micrographs taken from the top surface are presented in Fig. 4a-f, and the micrographs of magnified portion are presented in Fig. 4a-f (highlighted by dotted yellow box). It is clear from the micrographs that the smoothness of the surface decreases as the amount of HNT increases. The smoothest surface was observed for neat PLA (Fig. 4a), whereas the roughest surface morphology was observed for 7HNT (Fig. 4f). Several studies reported that the roughness of nanocomposite processed by FFF 3D printing increases with increase in filler content because of agglomeration tendency of fillers at the nozzle during 3D printing. This results in generation of a rough beads and hence an overall rough surface is created. Furthermore, HNT is relatively stiffer than melted PLA and PCL and this results in partial dragging of the polymer with the motion of nozzle while $3 \mathrm{D}$ printing. Although rough beads were observed in composite samples, the pore sizes and porosity remained almost the same in all the cases. Interconnected pores of different sizes were present ranging from $\sim 300-600 \mu \mathrm{m}$ in all the scaffolds.

The wettability of the scaffolds was measured using static water contact angle (WCA) method and the measurements obtained are presented in Fig. 5. The captured photographs of the droplets are shown on the top of the bar chart for all the samples tested. Overall, an increase in the surface wettability was observed with compositing. Upon addition of PCL into PLA, the contact angle decreased from $\sim 89^{\circ}$ (neat PLA) to $\sim 67^{\circ}$ (PLA/PCL), which was further decreased to $\sim 65^{\circ}$ with the addition of HNT (1HNT). A gradual increase in the contact angle was observed with increase in HNT content and it reached $\sim 85^{\circ}$ for $7 \mathrm{wt} \%$ loading of HNT (7HNT). This increase in the contact angle with increase in the HNT content is likely due to increase in surface roughness.

The mechanical properties were evaluated by performing tests on dogbone samples, and the representative stress-strain curves are shown in Fig. 6. The results obtained are summarized in Table 4 . The elastic modulus and tensile strength decrease with the addition of PCL into PLA as expected [44]. However, with the addition of HNT into PLA/PCL blend, both modulus and strength were recovered 
Figure 4 SEM images of the 3D printed samples: a Neat PLA, b PLA/PCL, c 1HNT, d $3 \mathrm{HNT}$, e $5 \mathrm{HNT}$ and $\mathbf{f} 7 \mathrm{HNT}$. The magnified portions of the bead surface are highlighted by yellow dotted boxes and corresponding micrographs are shown on the right.
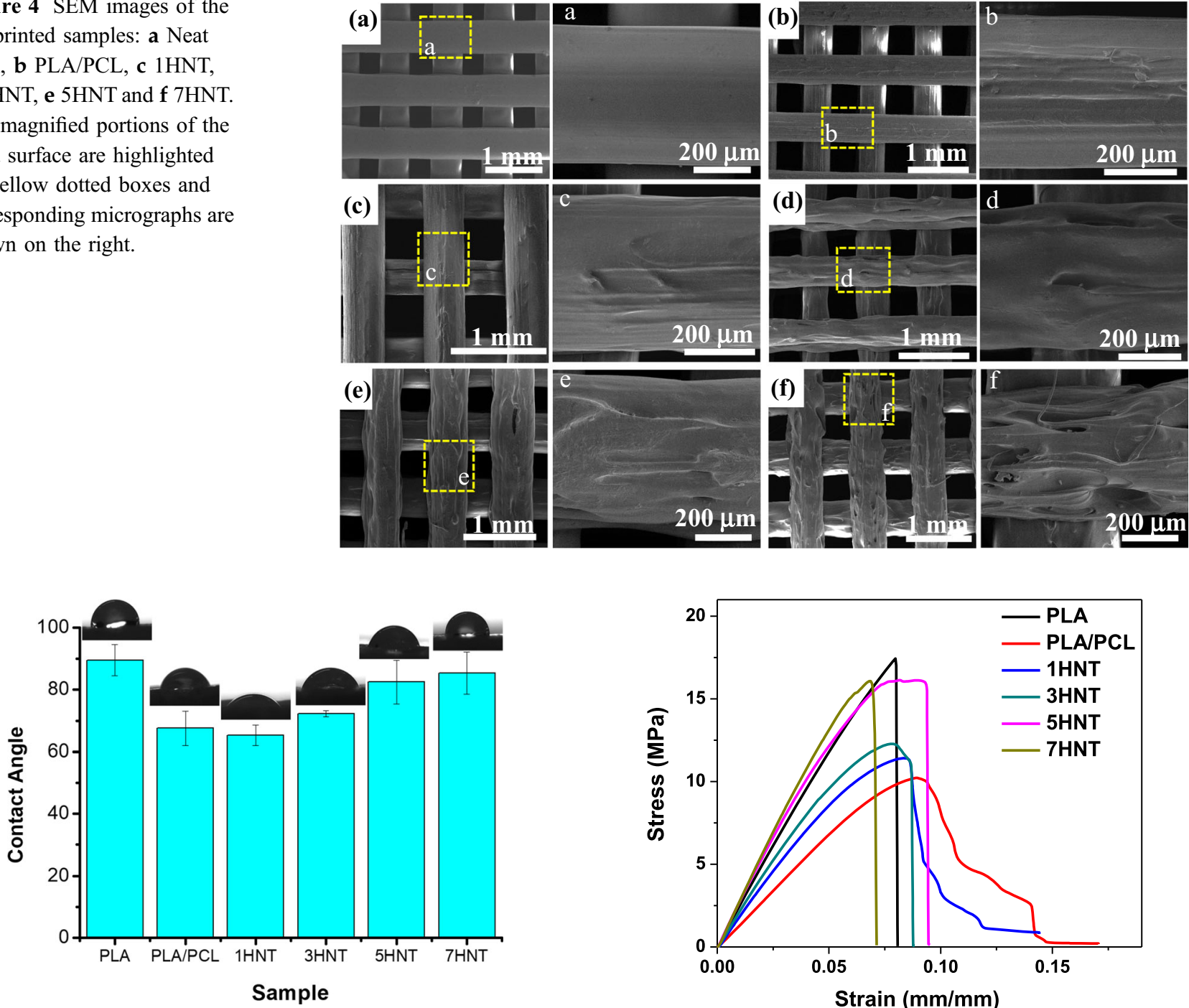

Figure 5 Surface wettability (contact angle measurements) of 3D printed samples.

[45]. PCL reduced the tensile strength but increased the elongation at break by $+99.58 \%$ (PLA/PCL) as compared to neat PLA. The increase in the modulus of PLA/PCL/HNT composites is due to the addition of stiffer HNTs into PLA/PCL blend while increase in the tensile strength of composites is due to effective load transfer from the blend to HNTs. The elongation at break of PCA/PCL/HNT composites decreases with increasing HNT content with a discrepancy at $5 \mathrm{wt} \% \mathrm{HNT}$. The increase in elongation of 5HNT sample is probably due to non-homogeneous dispersion of HNT in PLA/PCL blend $[45,46]$.

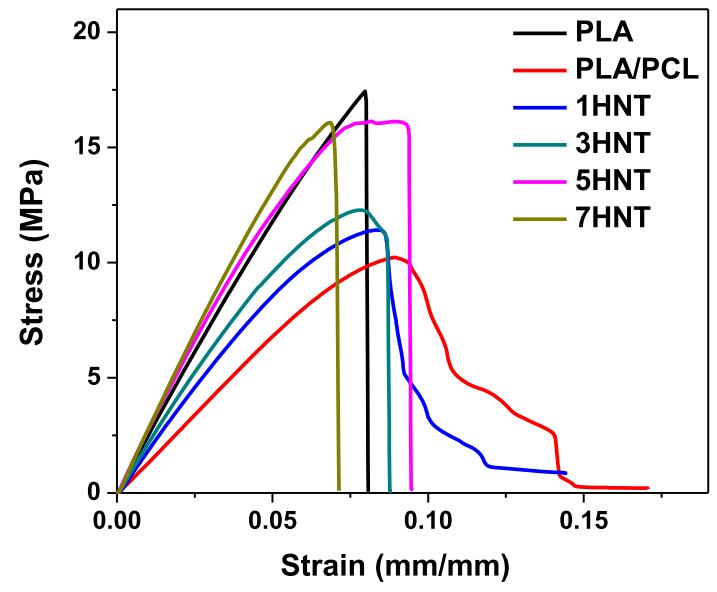

Figure 6 Representative stress-strain curves from tensile tests conducted on the dogbone samples.

\section{Biological characteristics of 3D printed scaffolds}

The bioactivity on the surface of the scaffolds was assessed in SBF and the apatite growth was analyzed using SEM. The micrographs are presented in Fig. 9. PCL and HNT both showed a positive effect on the bioactivity, and the bioactivity of the PLA remained unchanged. The surface morphology of the apatite growth observed from SEM showed increase in apatite growth with 3HNT, 5HNT and 7HNT (Fig. 7d-e) when compared to neat PLA (Fig. 7a). As can be clearly visualized, the size of apatite growth on these scaffolds increased with increase in HNT content. 
Table 4 Mechanical properties of PLA, PLA/PCL blend and PLA/PCL/HNT composite samples obtained from tensile tests

\begin{tabular}{lllc}
\hline Samples & Young's modulus $(\mathrm{MPa})$ & Tensile strength $(\mathrm{MPa})$ & Elongation at break $(\%)$ \\
\hline PLA & $246.56 \pm 5.53$ & $17.25 \pm 1.06$ & $7.18 \pm 1.01$ \\
PAL/PCL & $137.54 \pm 4.17$ & $10.40 \pm 0.59(-39.71 \%)$ & $14.33 \pm 0.84$ \\
$1 \mathrm{HNT}$ & $184.10 \pm 6.21$ & $11.45 \pm 0.61(-33.62 \%)$ & $12.3 \pm 0.65$ \\
$3 \mathrm{HNT}$ & $213.53 \pm 4.80$ & $12.87 \pm 0.54(-25.39 \%)$ & $8.53 \pm 0.67$ \\
$5 \mathrm{HNT}$ & $267.65 \pm 5.47$ & $15.52 \pm 1.12(-10.02 \%)$ & $9.37 \pm 0.50$ \\
7HNT & $281.19 \pm 3.90$ & $16.62 \pm 0.87(-3.65 \%)$ & $6.78 \pm 0.84$ \\
\hline
\end{tabular}
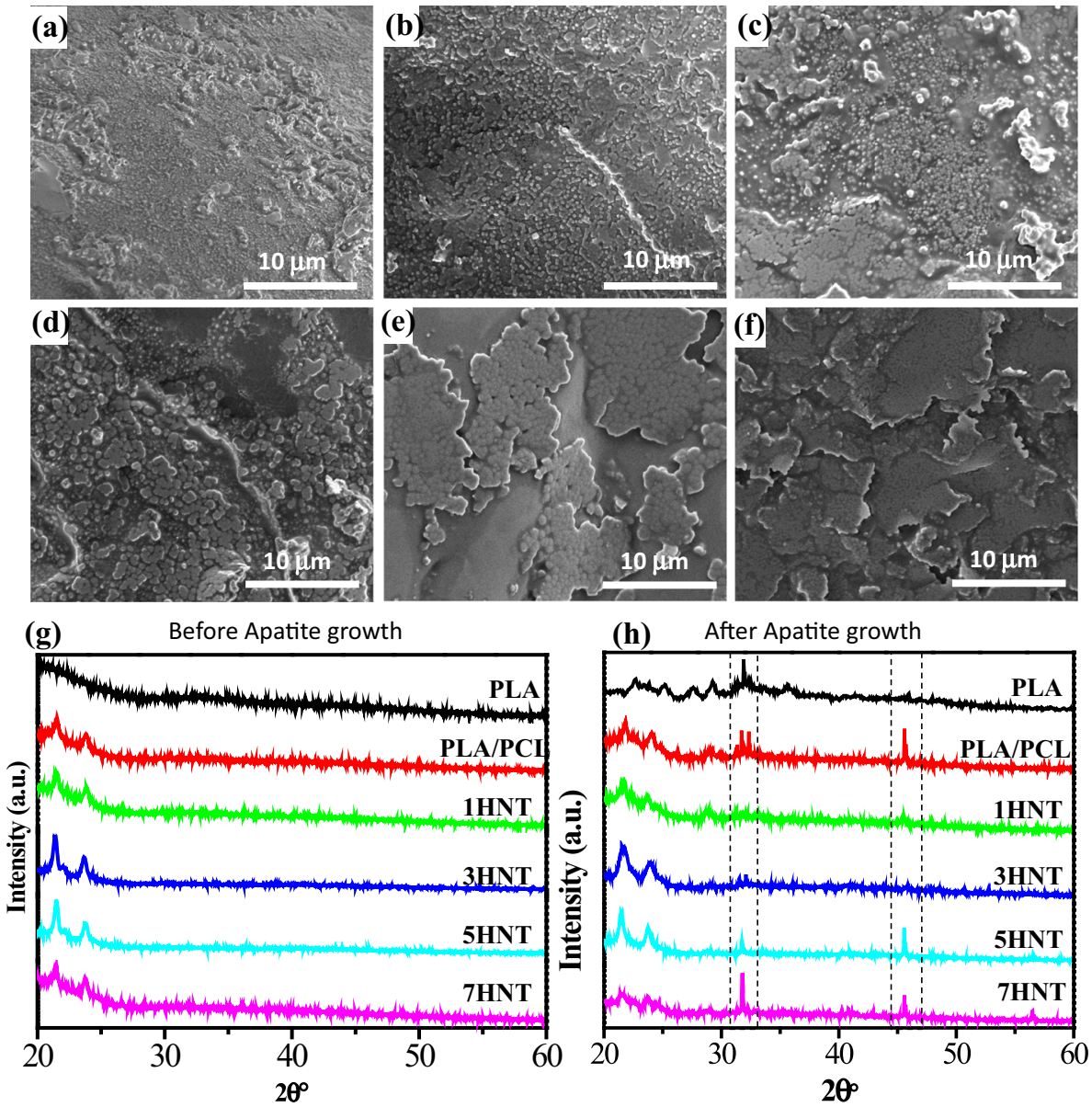

The fraction of apatite growth was quantified from XRD analysis and the XRD spectra obtained are presented in Fig. 7g and h. XRD analysis was performed before and after immersion tests and the obtained spectra were compared. The presence of extra peaks at $\sim 32.5^{\circ}$ and $\sim 46.2^{\circ}$ on the scaffolds after bioactivity test confirms the apatite (hydroxyapatite) growth [47] after immersion in SBF solution [48]. Integrating the area under the XRD apatite peaks, the fraction of apatite (\%) was calculated with respect to all peaks observed. Almost, the same fraction of apatite peaks was observed on PLA, PLA/
PCL and 1HNT scaffolds. A slight increase in bioactivity of $3 \mathrm{HNT}, 5 \mathrm{HNT}$ and $7 \mathrm{HNT}$ scaffolds was observed. The fraction of apatite growth increased from $5.4 \%$ (PLA) to $6.3 \%, 6.8 \%$ and $7.1 \%$ for $3 \mathrm{HNT}$, $5 \mathrm{HNT}$ and $7 \mathrm{HNT}$, respectively. Although surface wettability remained unchanged after HNT addition, the bioactivity increased, exhibiting the effect of HNT content. Note that, the effect of HNT reinforcement on bioactivity is dominant compared to the change in surface wettability.

In vitro biodegradation tests of the scaffolds were performed by immersing them in SBF for longer 

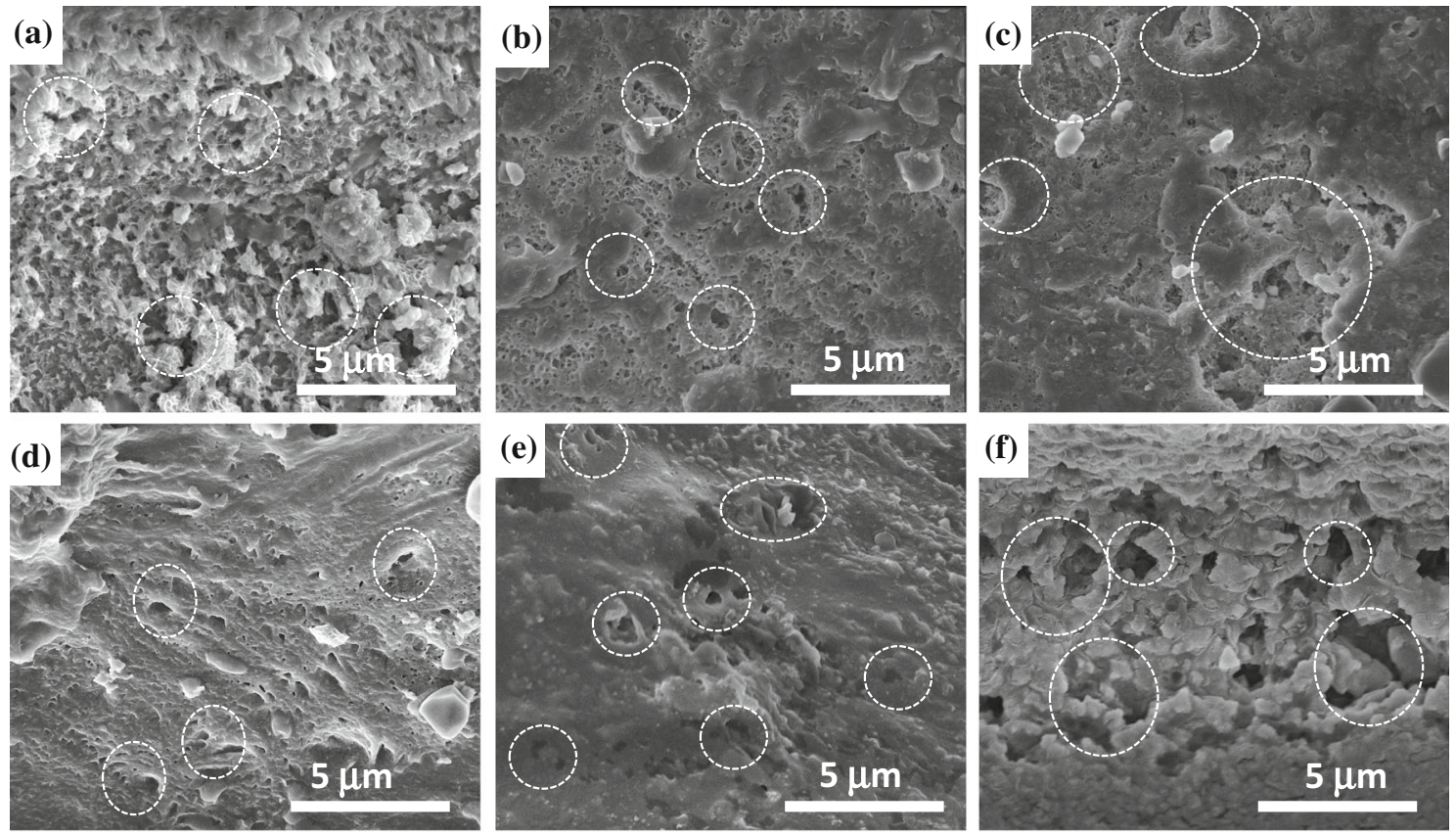

Figure 8 SEM images of the surfaces of samples after 4 weeks of degradation test. a Neat PLA, b PLA/PCL, c 1HNT, d 3HNT, e 5HNT and $\mathbf{f} 7 \mathrm{HNT}$. Dotted white circles show the pits and holes observed after the degradation test.

duration (up to 30 days) and the weight loss was calculated measuring the weights before and after the test. The surfaces of the scaffolds tested for biodegradation were observed under SEM and the micrographs are presented in Fig. 8. PCL demoted the rate of degradation of PLA as also evident from the literature that that PCL has a slower degradation rate than the PLA [9]. With the addition of PCL, the changes in the weight loss were reduced from $\sim 4.6$ to $\sim 1.3 \%$. The weight loss was further increased gradually to $\sim 4.4 \%$ (7HNT) as the HNT content was increased. The pits and holes formed during degradation on the surface of the scaffolds are observed under SEM and are highlighted in the micrographs by dotted white circles. As can be clearly seen from the images that neat PLA (Fig. 8a) has degraded the most among all scaffolds in line with weight loss measurements. The sizes of pits and holes are smaller in PLA/PCL scaffolds and this can be attributed to the slower rate of degradation of PCL as compared to PLA. The pits and holes increased in size and changed in shape as the content of HNT was increased. This can be correlated with the rough surface of the scaffolds and the subsequent longer exposure to SBF. The results from the degradation tests showed that the degradation rate can be tuned by changing the amount of HNT and PCL in PLA matrix.
When suspended, and not in physical contact with fibroblast culture, the degraded by-products from the fabricated scaffolds did not adversely affect the cells. On the contrary (Fig. 9a and b), when compared to control, there is a statistically significant increase in measured MTT absorbance from the by-product of PLA and a further significant increase is measured when PCL is added to the scaffold system. The various concentrations of HNT did not affect cellular metabolism as it is known for its biocompatibility $[49,50]$. Results from attachment test showed that cells were able to adhere and expand on the scaffolds as well (Fig. 9c-h). While 7HNT was the most hydrophobic, it is expected that attachment proteins from cell culture media would have been the most significant, and this supposedly will translate into higher cell attachment and proliferation. This is not the case, and it could be due to the size of the apatite that is deposited on the material of the scaffolds. Neat PLA has similar wettability as 7HNT, however, in the absence of apatite formation, showed equal fibroblast metabolism when compared to control. The reduction in HNT concentration showed reduced apatite formation and significant increment in measured MTT, which could indicate that surface topography is a factor here. Biologically, the fabricated scaffolds are biocompatible and support cellular attachment. 

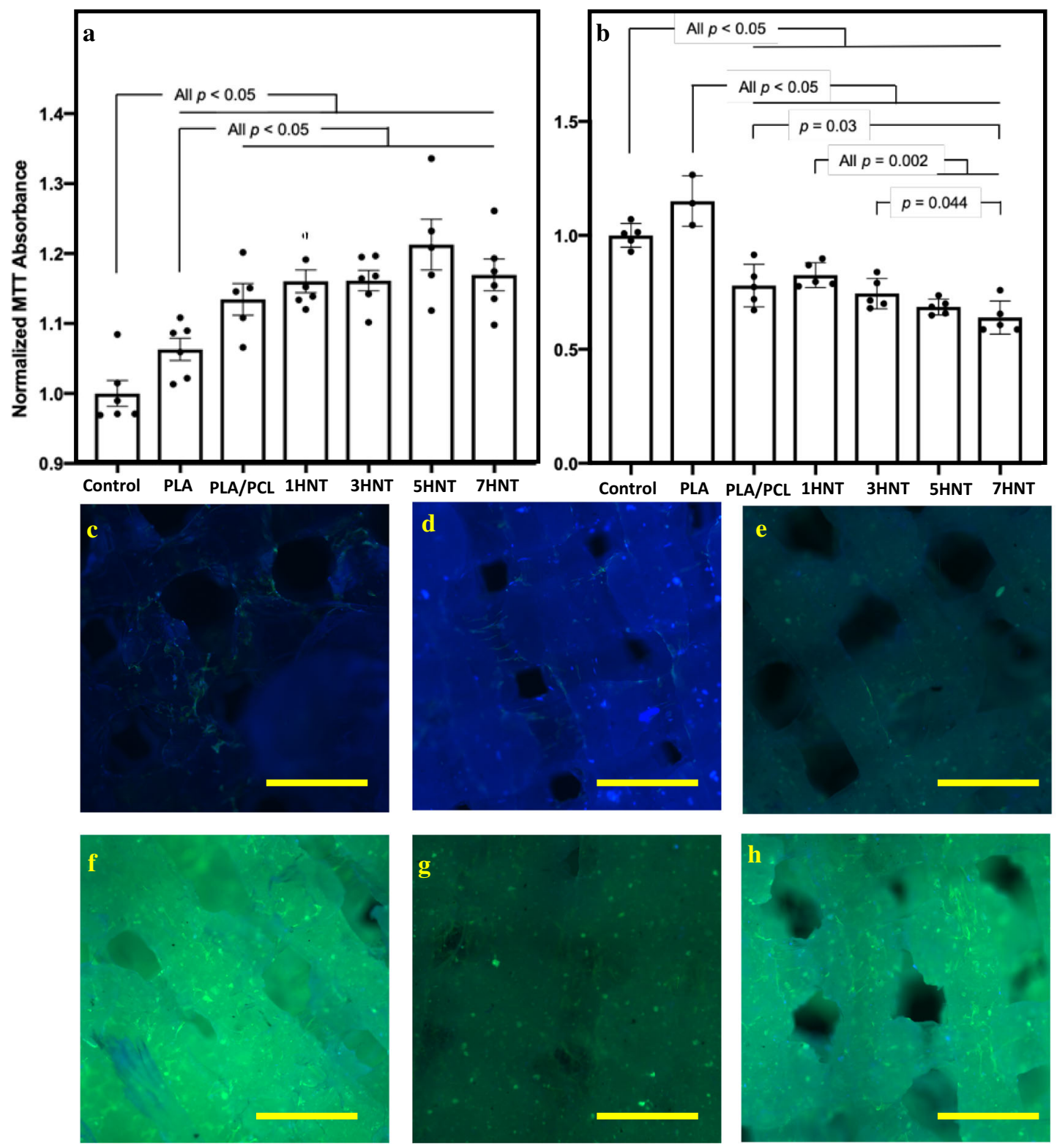

Figure 9 Biocompatibility of scaffolds with human dermal fibroblasts cells when normalized to control (tissue culture polystyrene): a Fibroblasts demonstrate increased metabolic activity when exposed to scaffolds (not in direct contact with the cells) and (b) the scaffolds are capable of supporting fibroblasts. Fluorescence microscopy of fibroblast cells attached on scaffolds.

\section{Conclusions}

The PLA/PCL/HNT composite filaments were developed by melt extrusion in conjunction with solvent casting. The scaffolds with $50 \%$ overall porosity were fabricated using FFF 3D printing. Thermal properties obtained from DSC showed a little decrease in the $T_{\mathrm{g}}$ with the addition of PCL into
There are no observable differences in fibroblast morphology on various fabricated scaffolds. Qualitatively, fibroblasts demonstrated equal elongations predominantly in the direction of scaffold fiber extrusion, which could be an indication of longitudinal surface artifacts: c Neat PLA, d PLA/PCL, e 1HNT, f $3 \mathrm{HNT}, \mathbf{g} 5 \mathrm{HNT}$ and $\mathbf{h} 7 \mathrm{HNT}$. The scale bar shown is $1 \mathrm{~mm}$.

PLA matrix while $T_{\mathrm{m}}$ remained unchanged with the addition of PCL and HNT. The surface wettability of scaffolds increased with the addition of PCL which then decreased slightly by the addition of HNT. This reduction in wettability with the addition of HNT could be attributed to increased surface roughness of printed scaffolds. All the scaffolds showed reasonable degree of bioactivity in SBF, demonstrating their 
potential for scaffold applications. PCL has reduced the degradation rate of the PLA and concomitantly reduced weight loss from 4.6 (PLA) to $1.3 \%$ (PLA/ PCL). PCL has improved the elongation while reducing the strength of PLA. The reduction in mechanical properties of PLA due to the addition of PCL was recovered by the incorporation of HNT into PLA/PCL blend. The reduction in HNT concentration showed reduced apatite formation and significant increment in measured MTT, which could indicate the influence of surface topography affected by the HNT. Biologically, the fabricated scaffolds are biocompatible and support cellular attachment. The amount of HNT and PCL can be optimally controlled to tune the biocompatibility and degradation rate of newly designed scaffolds. Furthermore, the 3D printed scaffolds showed adequate cytocompatibility and cell adhesion, demonstrating their potential for bone replacement and regeneration applications. To realize the full potential of PLA/PCL/HNT nanocomposites for scaffolds enabled by FFF 3D printing, the filament fabrication process needs to be optimized to ensure uniform dispersion of HNTs in PLA/PCL blend.

\section{Acknowledgements}

This publication is based upon work supported by the Khalifa University of Science and Technology under Award No. CIRA-2018-128. SK would like to thank the startup grant from University of Glasgow.

\section{Funding}

This study was funded by Abu Dhabi National Oil Company (ADNOC) (Award No: EX2016-000010).

\section{Declarations}

Conflict of interest The authors declare that they have no conflict of interest.

Open Access This article is licensed under a Creative Commons Attribution 4.0 International License, which permits use, sharing, adaptation, distribution and reproduction in any medium or format, as long as you give appropriate credit to the original author(s) and the source, provide a link to the Creative Commons licence, and indicate if changes were made. The images or other third party material in this article are included in the article's Creative Commons licence, unless indicated otherwise in a credit line to the material. If material is not included in the article's Creative Commons licence and your intended use is not permitted by statutory regulation or exceeds the permitted use, you will need to obtain permission directly from the copyright holder. To view a copy of this licence, visit http://creativecommons.org/licen ses/by/4.0/.

\section{References}

[1] Guo Y, Liang K, Ji Y (2019) New degradable composite elastomers of POC/PCL fabricated via in-situ copolymerization blending strategy. Eur Polym J 110:337-343

[2] Niza E et al (2020) PEI-coated PLA nanoparticles to enhance the antimicrobial activity of carvacrol. Food Chemi 328:127131

[3] Benatti ACB et al (2019) Bioreabsorbable polymers for tissue engineering: PLA, PGA, and their copolymers. Materials for biomedical engineering. Elsevier, pp 83-116

[4] Cerro D et al (2020) Effect of supercritical incorporation of cinnamaldehyde on physical-chemical properties, disintegration and toxicity studies of PLA/lignin nanocomposites. Int J Biol Macromol 167:255-266

[5] Farah S, Anderson DG, Langer R (2016) Physical and mechanical properties of PLA, and their functions in widespread applications: a comprehensive review. Adv Drug Deliv Rev 107:367-392

[6] Saini P, Arora M, Kumar MNVR (2016) Poly(lactic acid) blends in biomedical applications. Adv Drug Deliv Rev 107:47-59

[7] Kelnar I et al (2017) Graphite nanoplatelets-modified PLA/ PCL: effect of blend ratio and nanofiller localization on structure and properties. J Mech Behav Biomed Mater 71:271-278

[8] Kakroodi AR et al (2018) Facile production of biodegradable PCL/PLA in situ nanofibrillar composites with unprecedented compatibility between the blend components. Chem Eng J 351:976-984

[9] Ahmadzadeh Y, Babaei A, Goudarzi A (2018) Assessment of localization and degradation of $\mathrm{ZnO}$ nano-particles in the PLA/PCL biocompatible blend through a comprehensive rheological characterization. Polym Degrad Stab 158:136-147

[10] Zhou J et al (2004) Morphology and biodegradability of poly(E-caprolactone)/poly(vinyl alcohol) block copolymers. Polym J 36(9):695-704 
[11] Fortelny I et al (2019) Phase structure, compatibility and toughness of PLA/PCL blends: a review. Front Mater 6:206

[12] Hayashi T, Kanai H, Hayashi T (2001) Enzymatic degradation of poly( $\varepsilon$-caprolactone) fibers in vitro. Polym $\mathrm{J}$ 33(1):38-41

[13] Haq RHA, et al (2017) Characterization and mechanical analysis of PCL/PLA composites for FDM feedstock filament. In IOP conference series: materials science and engineering. IOP Publishing

[14] Nájera SE, Michel M, Kim N-S (2018) 3D Printed PLA/ $\mathrm{PCL} / \mathrm{TiO} 2$ composite for bone replacement and grafting. MRS Adv 3(40):2373-2378

[15] Alam $F$ et al (2020) Additively manufactured polyetheretherketone (PEEK) with carbon nanostructure reinforcement for biomedical structural applications. Adv Eng Mater 22(10):2000483

[16] Huang K et al (2019) Halloysite nanotube based scaffold for enhanced bone regeneration. ACS Biomater Sci Eng 5(8):4037-4047

[17] Setter OP, Segal E (2020) Halloysite nanotubes-the nano-bio interface. Nanoscale 12(46):23444-23460

[18] Long $Z$ et al (2017) Polyethyleneimine grafted short halloysite nanotubes for gene delivery. Mater Sci Eng C 81:224-235

[19] Yendluri R et al (2017) Application of halloysite clay nanotubes as a pharmaceutical excipient. Int $\mathrm{J}$ Pharm 521(1):267-273

[20] Liu M et al (2015) In vitro evaluation of alginate/halloysite nanotube composite scaffolds for tissue engineering. Mater Sci Eng C 49:700-712

[21] Luo Y, Humayun A, Mills DK (2020) Surface modification of 3D printed PLA/halloysite composite scaffolds with antibacterial and osteogenic capabilities. Appl Sci 10(11):3971

[22] Humayun A, Luo Y, Elumalai A, Mills DK (2020) 3D printed antimicrobial PLA constructs functionalised with zinc-coated halloysite nanotubes-Ag-chitosan oligosaccharide lactate. Mater Technol 1-8. https://doi.org/10.1080/106 67857.2020.1806188

[23] Weisman JA et al (2017) Doped halloysite nanotubes for use in the $3 \mathrm{D}$ printing of medical devices. Bioengineering 4(4):96

[24] Wu F et al (2019) Halloysite nanotubes coated 3D printed PLA pattern for guiding human mesenchymal stem cells (hMSCs) orientation. Chem Eng J 359:672-683

[25] Panjwani MB, et al (2020) 10: performance of 3D printed poly(lactic acid)/halloysite nanocomposites. In: Goh KL (eds) Interfaces in particle and fibre reinforced composites. Woodhead Publishing, pp. 251-267
[26] Arif MF et al (2018) Performance of biocompatible PEEK processed by fused deposition additive manufacturing. Mater Des 146:249-259

[27] Dugbenoo E et al (2018) Enhanced bonding via additive manufacturing-enabled surface tailoring of 3D printed continuous-fiber composites. Adv Eng Mater 20(12):1800691

[28] Rajkumar AR, Shanmugam K (2018) Additive manufacturing-enabled shape transformations via FFF 4D printing. J Mater Res 33(24):4362-4376

[29] Ligon SC et al (2017) Polymers for 3D printing and customized additive manufacturing. Chem Rev 117(15):10212-10290

[30] Ubaid J, Wardle BL, Kumar S (2018) Strength and performance enhancement of multilayers by spatial tailoring of adherend compliance and morphology via multimaterial jetting additive manufacturing. Sci Rep 8(1):1-10

[31] Capel AJ et al (2018) 3D printing for chemical, pharmaceutical and biological applications. Nat Rev Chem 2(12):422-436

[32] Arif $\mathrm{M}$ et al (2020) Multifunctional performance of carbon nanotubes and graphene nanoplatelets reinforced PEEK composites enabled via FFF additive manufacturing. Compos Part B: Eng 184:107625

[33] Gomez-Gras G et al (2018) Fatigue performance of fused filament fabrication PLA specimens. Mater Des 140:278-285

[34] Kazmer D (2017) 28-three-dimensional printing of plastics. In: Kutz M (ed) Applied plastics engineering handbook. Second Edition. William Andrew Publishing, pp. 617-634

[35] Krieger KJ et al (2019) Simple and customizable method for fabrication of high-aspect ratio microneedle molds using low-cost 3D printing. Microsyst Nanoeng 5(1):1-14

[36] Andrew JJ, Verma P, Kumar S (2021) Impact behavior of nanoengineered, 3D printed plate-lattices. Mater Des 202:109516

[37] Gupta V et al (2021) Additive manufacturing enabled, microarchitected, hierarchically porous polylactic-acid/ Lithium iron phosphate/carbon nanotube nanocomposite electrodes for high performance Li-Ion batteries. J Power Sour 494:229625

[38] Verma P, Schiffer A, Kumar S (2021) Thermo-resistive and thermo-piezoresistive sensitivity of carbon nanostructure engineered thermoplastic composites processed via additive manufacturing. Polym Test 93:106961

[39] Slouf $M$ et al (2020) Monitoring of morphology and properties during preparation of PCL/PLA microfibrillar composites with organophilic montmorillonite. Front Mater $7: 188$ 
[40] Alam F et al (2020) Microarchitected 3D printed polylactic acid (PLA) nanocomposite scaffolds for biomedical applications. J Mech Behav Biomed Mater 103:103576

[41] Alam F, Varadarajan KM, Kumar S (2020) 3D printed polylactic acid nanocomposite scaffolds for tissue engineering applications. Polym Test 81:106203

[42] Chozhanathmisra M et al (2019) Halloysite nanotube-reinforced ion-incorporated hydroxyapatite-chitosan composite coating on Ti-6Al-4 V alloy for implant application. J Chem 2019:7472058

[43] Jing $\mathrm{H}$ et al (2014) Preparation and characterization of polycarbonate nanocomposites based on surface-modified halloysite nanotubes. Polym J 46(5):307-312

[44] Robeson LM (2007) Polymer blends. In: Robeson LM (ed) A Comprehensive review. Carl Hanser Verlag GmbH \& Co. KG, München

[45] Dong Y et al (2015) Polylactic acid (PLA)/halloysite nanotube (HNT) composite mats: influence of HNT content and modification. Compos A Appl Sci Manuf 76:28-36
[46] Venkatesh C et al (2019) Additive manufacturing of PLA/ HNT nanocomposites for biomedical applications. Procedia Manuf 38:17-24

[47] Alam F et al (2015) Processing, characterization and fretting wear of zinc oxide and silver nanoparticles reinforced ultra high molecular weight polyethylene biopolymer nanocomposite. Jom 67(4):688-701

[48] Bohner M, Lemaitre J (2009) Can bioactivity be tested in vitro with SBF solution? Biomaterials 30(12):2175-2179

[49] Satish S, Tharmavaram M, Rawtani D (2019) Halloysite nanotubes as a nature's boon for biomedical applications. Nanobiomedicine 6:1849543519863625

[50] Santos AC et al (2018) Halloysite clay nanotubes for life sciences applications: From drug encapsulation to bioscaffold. Adv Coll Interface Sci 257:58-70

Publisher's Note Springer Nature remains neutral with regard to jurisdictional claims in published maps and institutional affiliations. 